\title{
Prevalence and molecular identification of Cryptosporidium ISOLATES FROM PET LIZARDS AND SNAKES IN ITALY
}

\author{
RINALDI L.*, CAPASSO M.*, MIHALCA A.D.**, CIRILLO R.*, CRINGOLI G.* \& CACCIÒ S.***
}

\section{Summary:}

In order to acquire prevalence and genetic data on Cryptosporidium infections in captive lizards and snakes kept as pets, a survey was conducted on 150 individual reptiles from southern Italy. Fecal samples were preserved in $5 \%$ formalin and analyzed using a commercial immunofluorescence assay (IFA) for the detection of Cryptosporidium oocysts and Giardia cysts. IFA revealed the presence of Cryptosporidium oocysts in nine of the 150 samples examined $(6.0 \%)$, precisely in $6 / 125$ snakes $(4.8 \%)$ and in $3 / 25$ lizards (12.0\%); all fecal samples tested negative for the presence of Giardia cysts. Molecular characterization based on nested PCR amplification and sequencing of the SSU-rRNA gene, revealed the presence of Cryptosporidium serpentis in three samples from snakes (Boa constrictor constrictor, Elapheguttata guttata guttata and Python molurus).

KEY WORDS: Cryptosporidium, reptile, lizard, snake, Python molurus, Italy.

\footnotetext{
I
} $\mathrm{n}$ recent years, the number of reptiles kept as exotic pets in households has significantly increased (Copping, 2008). However, the acquisition of reptiles, either as captive bred or wild caught animals, yields a high risk of disease introduction into a collection and for this reason, reptilian medicine has evolved greatly in recent decades (Pasmans et al., 2008). Stressful life, concentration of animals and the presence of different subjects in a small living space can promote the spreading of pathogens. Moreover, all these factors may suppress the immune response in reptiles and increase the opportunity for pathogens to cause infections and consequent diseases (Rataj et al., 2011; Dipineto et al., 2012) which can be spread to other individuals or

\footnotetext{
* Department of Animal Pathology and Health, Faculty of Veterinary Medicine, University of Naples Federico II, Via della Veterinaria 1, 80137 Naples, Italy.

** Department of Parasitology and Parasitic Diseases, Faculty of Veterinary Medicine, University of Agricultural Sciences and Veterinary Medicine Cluj-Napoca, Calea Manastur 3-5, Cluj-Napoca 400372, Romania.

*** Department of Infectious, Parasitic and Immunomediated Diseases, Istituto Superiore di Sanità, viale Regina Elena 299, 00161 Rome, Italy.

Correspondence: Laura Rinaldi.

Tel.: 390812536281 - Fax: +390812536282

E-mail: lrinaldi@unina.it - URL: http://www.parassitologia.unina.it
}

Résumé : PRÉVAlenCE ET IDENTIFICATION PAR GÉNOTYPAGE DE CRYPTOSPORIDIUM ISOLÉS DE LÉZARDS ET DE SERPENTS, ANIMAUX DE COMPAGNIE, EN ITALIE

Afin de déterminer la prévalence et l'identification par génotypage des infections à Cryptosporidium chez les lézards et les serpents, animaux de compagnie, une enquête a été menée en Italie du sud sur 150 reptiles. Les échantillons fécaux ont été conservés dans du formol à $5 \%$ et analysés à l'aide d'un test d'immunofluorescence (IF) du commerce, permettant la détection simultanée d'oocystes de Cryptosporidium et de kystes de Giardia. L'IF a montré la présence d'oocystes de Cryptosporidium dans neuf des 150 échantillons examinés $(6,0 \%)$, plus précisément chez $6 / 125$ serpents $(4,8 \%)$ et $3 / 25$ lézards (12,0\%). Tous les échantillons ont été négatifs pour la recherche des kystes de Giardia. La caractérisation moléculaire, basée sur l'amplification par PCR nichée et séquençage du gène SSU-rRNA a identifié C. serpentis dans trois échantillons fécaux de serpents (Boa constrictor constrictor, Elaphe guttata guttata et Python molurus).

MOTS-CLÉS : Cryptosporidium, reptile, lézard, serpent, Python molurus, Italie.

other species, including humans. Therefore, an examination for endoparasites should be performed as part of the routine check of all reptiles and only parasitefree or successfully treated animals should be introduced into a healthy collection (Pasmans et al., 2008). Cryptosporidium infections are common in wild and captive reptiles (Upton et al., 1989) and often establish as chronic and sometimes lethal infections (Pasmans et al., 2008). Several Cryptosporidium species have been described from reptiles since the first report by Brownstein (1977). However, currently only two species are considered valid, namely $C$. serpentis and $C$. varanii (Fayer, 2008). C. serpentis is associated mainly with snakes and infects usually the gastric epithelium while $C$. varanii has intestinal predilection and is primarily associated with lizards (Xiao et al., 2004). Recently, molecular evidence suggested the existence of a third species in tortoises (Traversa, 2010).

Snakes infected with $C$. serpentis may show symptoms of mild to severe gastritis with frequent regurgitation, particularly after feeding, while $C$. varanii causes enteritis and diarrhea. None of this reptile associated Cryptosporidium species is infective to mammals; hence their zoonotic importance is absent. However, oocysts of the zoonotic species C. parvum and C. muris have 
been also detected in the feces of captive snakes and lizards, and probably originate from infected mammals used as food by carnivorous reptiles (Xiao et al., 2004; Pedraza-Diaz et al., 2009; Richter et al., 2011). The bovine genotype of $C$. parvum has been recently isolated also from wild tortoises in Romania (D'Amico et al., unpublished data).

Despite the importance of Cryptosporidium infections in snakes and lizards, due to their impact on animal and public health and welfare, only few comprehensive surveys have been conducted on the prevalence and molecular characterization of cryptosporidium isolates in captive reptiles (e.g. Pedraza-Diaz et al., 2009; Richter et al., 2011).

In this study, we performed a survey on the prevalence and molecular identification of Cryptosporidium in fecal samples from reptiles (snakes and lizards) kept as pets in the Campania region of southern Italy.

\section{MATERIALS AND METHOD}

\section{SAMPLING AND IMMUNOFLUORESCENCE}

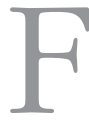
rom September 2009 to June 2010 individual fecal samples and cloacal swabs were collected from 150 captive reptiles (125 snakes and 25 lizards). Out of these 150 animals, 94 (62.7\%) were housed in exotic animal farms, nine $(6 \%)$ in pet shops and $47(31.3 \%)$ in private households. For each animal, anamnesis data (species, sex, age, micro-environment conditions, captive-born or wild-caught origin, feeding, cohabitation, gastro-intestinal symptoms, use of antiparasitic treatments) were recorded. All fecal samples were stored in $5 \%$ formalin and subsequently examined in the laboratory using the FLOTAC Pellet Technique (Cringoli et al., 2010) in order to detect parasitic and pseudoparasitic elements (for the results see Rinaldi et al., 2012). In addition, on each fecal sample, a direct immunofluorescence assay (IFA) was used to investigate the presence of Cryptosporidium oocysts and Giardia cysts using a commercial kit (MERIFLUOR Cryptosporidium/Giardia, Meridian Bioscience Diagnostic, Cincinnati, OH, USA). Fecal suspension was concentrated with a Sucrose Solution (specific gravity $=1.200)$ and the sediment obtained was applied to slides for IF and stained according to the manufacturer's instructions. Oocysts were identified and counted by fluorescence microscopy at $400 \times$ on the basis of apple-green fluorescence and morphometric features (size, width and internal structures).

\section{MOLECULAR CHARACTERIZATION}

Prior to DNA extraction, fecal samples (200 $\mu \mathrm{l})$ resulted positive for Cryptosporidium by IFA were washed three times with phosphate-buffered saline $(\mathrm{pH} 7.4)$ and centrifuged. DNA extraction was performed using a commercial kit (QIAamp DNA stool mini kit, Qiagen, Hilden, Germany).

A nested PCR protocol (Nichols et al., 2003) was used for the amplification of a fragment of the small subunit ribosomal RNA gene (SSU-rRNA) of Cryptosporidium spp. In the primary PCR, a fragment of $\sim 655 \mathrm{bp}$ was amplified with the primers N-DIAGF2 (5'-CAA TTG GAG GGC AAG TCT GGT GCC AGC-3') and NDIAGR2 (5'-CCT TCC TAT GTC TGG ACC TGG TGA GT-3'), whereas in the nested PCR reaction, a fragment of $\sim 455$ bp was amplified using the primers DIAGF (5'-AAG CTC GTA GTT GGA TTT CTG-3') and DIAGR (5'-TAA GGT GCT GAA GGA GTA AGG-3') originally described by Johnson et al. (1995). PCR conditions were as reported by Nichols et al. (2003). Amplification was performed in a Verity96 thermal cycler. Negative (water) and positive (DNA of C. parvum) controls were included in each experiment. Amplification products were separated by gel electrophoresis and visualized by ethidium bromide staining.

PCR products were purified using the Qiaquick purification kit (Qiagen, Milan, Italy) and sequenced on both strands using the ABI Prism BIGDYE Terminator Cycle Sequencing Kit (Life Technologies, Carlsbad, CA, USA) according to the manufacturer's instructions. The sequencing reactions were analyzed using the $\mathrm{ABI}$ 3100 automatic sequencer (Applied Biosystems) and sequences were assembled using the software program SeqMan II (DNASTAR, Madison WI, USA).

\section{RESULTS}

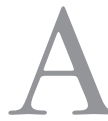
11 the 150 reptiles in this study were asymptomatic and didn't receive any antiparasitic treatment. The diet of snakes included rodents, chickens and rabbits; lizards were fed mainly with vegetables and arthropods, depending on the species and size. A total of 125 snakes of 16 different genera (Acanthophis, Aspidites, Boa, Bogertophis, Crotalus, Drymarcon, Elaphe, Gonyosoma, Hydrodynastes, Lampropeltis, Lamprophis, Lichanura, Morelia, Oreocryptophis, Python, Rhyncophis) and 25 lizards of ten different genera (Basiliscus, Chamaleo, Clamydiosaurus, Eublepharis, Iguana, Phelsuma, Physignatus, Pogona, Tupinambis, Zoonosaurus) were examined.

The presence of Cryptosporidium oocysts was detected by IFA in nine out of the 150 samples examined $(6.0 \% ; 95 \% \mathrm{CI}=3.0-11.4 \%)$, precisely in $6 / 125$ snakes $(4.8 \% ; 95 \% \mathrm{CI}=2.0-10.6 \%)$ and in $3 / 25$ lizards $(12.0 \%$; $95 \% \mathrm{CI}=3.1-32.3 \%)$ (Table I). In general, the number of oocysts observed was low, 


\begin{tabular}{|c|c|c|c|c|c|}
\hline \multirow[b]{2}{*}{ Group } & \multirow[b]{2}{*}{ Common name } & \multirow[b]{2}{*}{ Scientific name } & \multicolumn{3}{|c|}{ Positivity to Cryptosporidium } \\
\hline & & & IF & PCR & Cryptosporidium species \\
\hline \multirow[t]{3}{*}{ Lizards } & Veiled chameleon & Chamaeleo calyptratus & 1 & 1 & n.d. \\
\hline & Frilled lizard & Chlamydosaurus kingii & 1 & 1 & n.d. \\
\hline & Leopard gecko & Eublepharis macularius & 1 & n.a. & - \\
\hline \multirow[t]{4}{*}{ Snakes } & Boa constrictor & Boa constrictor constrictor & 1 & 1 & C. serpentis \\
\hline & Boa constrictor & Boa constrictor imperator & 1 & n.a. & - \\
\hline & Corn snake & Elaphe guttata guttata & 2 & 1 & C. serpentis \\
\hline & Indian rock python & Python molurus & 2 & 1 & C. serpentis \\
\hline
\end{tabular}

n.a. $=$ not available for molecular characterization; n.d. $=$ not identified at the species level.

Table I. - List of the reptile species that tested positive by IFA for the presence of Cryptosporidium oocysts.

with most samples showing between one and ten oocysts per slide. All fecal samples tested negative for the presence of Giardia cysts. No significant association ( $\mathrm{P}>0.05$ using the chi-squared test) was found between the origin of the reptiles (exotic farms, pets shop or private households) and the positivity to Cryptosporidium.

Only five of the nine Cryptosporidium-positive samples were available for molecular characterization. Nested PCR amplification of the SSU-rRNA gene yielded the expected fragment ( $450 \mathrm{bp}$ ) from three snake isolates (Boa constrictor constrictor, Elaphe guttata guttata, Python molurus) and sequencing revealed $100 \%$ homology with C. serpentis (e.g., GenBank accession number AF151376). In the case of lizards, weak amplification products were observed after the first PCR, but no amplification was obtained using nested PCR. The sequences of the first amplification products were of insufficient quality to identify the species.

\section{DISCUSSION}

7 ryptosporidium infections are common in wild and captive reptiles; over 80 reptile species have been reported to be infected, including snakes, lizards and tortoises (Graczyk, 2008). The present study reports the results of the first IFA and molecular survey of Cryptosporidium in snakes and lizards in Italy. A recent coprological survey in pet reptiles in Italy (Papini et al., 2011) showed Cryptosporidium infection in two leopard geckos (Eublepharis macularius) but molecular identification of the Cryptosporidium isolates to species level was not performed. In this study the presence of Cryptosporidium oocysts was detected by IFA in 9 samples (6.0\%), six from snakes and three from lizards. Molecular characterization by nested PCR amplification and sequencing of the SSU-rRNA gene demonstrates the presence of $C$. serpentis in the snakes Boa constrictor, Elaphe guttata guttata and Python molurus. It should be noted that C. serpentis have been previously reported in boa and corn snakes, however, according to the author's knowledge, this is the first report of the presence of Cryptosporidium (C. serpentis) in Python molurus (Indian rock python). On the other hand, the Cryptosporidium parasites present in samples from lizards could not be identified by this approach. The weak amplification observed after the primary PCR reaction, and the lack of specific amplification in the secondary PCR reaction was mainly due to the preservation of the samples in formalin (see below) but could also suggest that DNAs from other organisms can bind PCR primers and reduce or prevent amplification of Cryptosporidium DNA.

Regarding Giardia, the present study confirmed the absence of this protozoan in snakes and lizards. Indeed, reptiles are not listed as potential hosts for parasites in the Giardia genus (Thompson et al., 2011) and only one description of a Giardia varani-like flagellate from a water monitor, Varanus salvator, has been reported in Malaysia (Upton et al., 1997).

This study added data to the few comprehensive surveys on the prevalence of Cryptosporidium in pet snakes and lizards in Europe (e.g. Pedraza-Diaz et al., 2009; Richter et al., 2011). In agreement with previous studies (i.e. Pedraza-Diaz et al., 2009; Papini et al., 2011), none of the infected animals showed signs of clinical cryptosporidiosis at the sampling time. The low numbers of oocysts detected as well as the absence of clinical signs may indicate a subclinical stage of infection, which can last for years in these animals (Pasmans et al., 2008). Even if Cryptosporidium infection in reptiles is frequently chronic, C. serpentis infection in snakes may cause clinical disease (gastric hyperplasia, postprandial regurgitation and firm midbody swelling or chronic debilitating enteritis) and pathological changes. Currently, there are no effective control strategies against cryptosporidiosis in reptiles (Plutzer et al., 2007). 
C. varanii is the species most frequently detected in lizards causing weight loss and enteritis in leopard geckos, Eublepharis macularius (Taylor et al., 1999; Terrell et al., 2003; Deming et al., 2008); in addition, cloacal prolapse and cystitis in green iguana (Iguana iguana) caused by a novel Cryptosporidium genotype has been also recently described (Kik et al., 2011).

The aim of this survey was to obtain preliminary data on the prevalence of cryptosporidium infection in snakes and lizards kept as pets in southern Italy; unfortunately, identification of Cryptosporidium species could not be obtained for all the IFA positive samples, probably because feces were fixed in formalin and this has an inhibitory effect on PCR amplification. Further studies deserve to be carried out in order to: (i) improve molecular identification; (ii) understand the source of Cryptosporidium infection for snakes and lizards; (iii) study in depth the real pathogenic effect of C. serpentis, C. varanii and other species; (iv) verify the possible occurrence of zoonotic Cryptosporidium species (as parasites or pseudo-parasites) in these new exotic pets.

\section{REFERENCES}

Brownstein D.G., Stranberg J.D., Montali R.J., Bush M. \& FORTNER J. Cryptosporidium in snakes with hypertrophic gastritis. Veterinary Pathology, 1977, 14, 606-617.

Copping J. Reptiles now more popular pets than dogs. The Telegraph, 2008, 22 November.

Cringoli G., Rinaldi L., Maurelli M.P. \& Utzinger J. FlOtaC: new multivalent techniques for qualitative and quantitative copromicroscopic diagnosis of parasites in animals and humans. Nature Protocols, 2010, 5, 503-515.

Deming C., Greiner E. \& Uhl E.W. Proliferative enteritis in leopard geckos (Eublepharis macularius) associated with Cryptosporidium sp. infection. Journal of Zoo and Wildlife Medicine, 2008, 39, 600-607.

Dipineto L., Capasso M., Maurelli M.P., Russo T.P., Pepe P., Capone G., Fioretti A., Cringoli G. \& Rinaldi L. Survey of co-infection by Salmonella and oxyurids in tortoises. BMC Veterinary Research, 2012, accepted.

FAYER R. General biology, in: Cryptosporidium and Cryptosporidiosis, $2^{\text {nd }}$ edition. Fayer R. \& Xiao L. (eds), CRC Press, Boca Raton, Florida, 2008, 1-42.

GraczyK T.K. Fish, Amphibians, and Reptiles, in: Cryptosporidium and Cryptosporidiosis, $2^{\text {nd }}$ edition. Fayer R. \& Xiao L. (eds), CRC Press, Boca Raton, Florida, 2008, 387-394.

Johnson D.W., Pieniazek N.J., Griffin D.W., Misener L. \& Rose J.B. Development of a PCR protocol for sensitive detection of Cryptosporidium oocysts in water samples. Applied and Environmental Microbiology, 1995, 61, 3849-3855.

Kik M.J., van Asten A.J., Lenstra J.A. \& Kirpensteijn J. Cloaca prolapse and cystitis in green iguana (Iguana iguana) caused by a novel Cryptosporidium species. Veterinary Parasitology, 2011, 175, 165-167.
Nichols R.A., Campbell B.M. \& SMith H.V. Identification of Cryptosporidium spp. oocysts in United Kingdom noncarbonated natural mineral waters and drinking waters by using a modified nested PCR-restriction fragment length polymorphism assay. Applied and Environmental Microbiology, 2003, 69, 4183-4189.

Papini R., ManetTi C. \& Mancianti F. Coprological survey in pet reptiles in Italy. Veterinary Record, 2011, 169, 207.

Pasmans F., Blahak S., Martel A. \& Pantchev N. Introducing reptiles into a captive collection: the role of the veterinarian. Veterinary Journal, 2008, 175, 53-68.

Pedraza-Díaz S., Ortega-Mora L.M., Carrión B.A., Navarro V. \& Gómez-Bautista M. Molecular characterisation of Cryptosporidium isolates from pet reptiles. Veterinary Parasitology, 2009, 204-210.

Plutzer J. \& Karanis P. Molecular identification of a Cryptosporidium saurophilum from corn snake (Elaphe guttata guttata). Parasitology Research, 2007, 101, 1141-1145.

Rataj A.V., Lindtner-Knific R., Vlahović K., Mavri U. \& DovČ A. Parasites in pet reptiles. Acta Veterinaria Scandinavica, 2011, 53, 33.

Richter B., Nedorost N., Maderner A. \& Weissenböck H. Detection of Cryptosporidium species in feces or gastric contents from snakes and lizards as determined by polymerase chain reaction analysis and partial sequencing of the 18S ribosomal RNA gene. Journal of Veterinary Diagnostic Investigation, 2011, 23, 430-435.

Rinaldi L., Mihalca A.D., Cirillo R., Maurelli M.P., Montesano M., Capasso M. \& Cringoli G. FlOTAC can detect parasitic and pseudoparasitic elements in reptiles. Experimental Parasitology, 2012, 130, 282-284.

Taylor M.A., Geach M.R. \& CoOley W.A. Clinical and pathological observations on natural infections of cryptosporidiosis and flagellate protozoa in leopard geckos (Eublepharis macularius). Veterinary Parasitology, 1999, 145, 695-699.

Terrell S.P., Uhl E.W. \& Funk R.S. Proliferative enteritis in leopard geckos (Eublepharis macularius) associated with Cryptosporidium sp. infection. Journal of Zoo and Wildlife Medicine, 2003, 34, 69-75.

Thompson R.C. \& Smith A. Zoonotic enteric protozoa. Veterinary Parasitology, 2011, 182, 70-78

Traversa D. Evidence for a new species of Cryptosporidium infecting tortoises: Cryptosporidium ducismarci. Parasites \& Vectors, 2010, 3, 21.

Upton S.J., McAllister C.T., Freed P.S. \& Barnard S.M. Cryptosporidium spp. in wild and captive reptiles. Journal of Wildlife Disease, 1989, 25, 20-30.

Upton S.J. \& ZIEN C.A. Description of a Giardia varani-like flagellate from a water monitor, Varanus salvator, from Malaysia. Journal of Parasitology, 1997, 83, 970-971.

Xiao L., Ryan U.M., Graczyk T.K., Limor J., Li L., Kombert M., Junge R., Sulaiman I.M., Zhou L., Arrowood M.J., Koudela B., Modrý D. \& Lal A.A. Genetic diversity of Cryptosporidium spp. in captive reptiles. Applied and Environmental Microbiology, 2004, 70, 891-899.

Received on May $8^{\text {th }}, 2012$ Accepted on July $4^{\text {th }}, 2012$ 